\title{
The British Intestinal Failure Survey (BIFS): A referral registry to record and determine the outcome of childhood intestinal failure
}

\author{
H. Gowen ${ }^{1}$ and C. Lloyd ${ }^{2}$ \\ ${ }^{1}$ British Intestinal Failure Survey, 3rd Floor Registry Office, Institute of Child Health, Whittall Street, Birmingham B4 6NH, \\ UK and ${ }^{2}$ Liver Unit, Birmingham Children's Hospital, Steelhouse Lane, Birmingham B4 6NH, UK
}

Intestinal failure (IF) is a complex, life-threatening disorder requiring highly-specialised treatment and with great variation in outcome. Most patients recover, many require continuing support on home parenteral nutrition and a proportion may progress to intestinal transplantation (ITx). The paucity of information in the UK about incidence causes and outcome of IF has impeded rational planning of longterm clinical services including ITx.

BIFS attempts to prospectively identify all cases of IF in infants and children in the UK, including hospitalised children, using parenteral nutrition (PN) dependency for $28 \mathrm{~d}$ as the inclusion criterion.

Initially, a pilot study in six centres enrolled all children $<16$ years of age at the start of PN. Since July 2007 twenty-five centres identified via the British Society for Paediatric Gastroenterology, Hepatology and Nutrition membership list have registered an interest in participating and ten have registered patients. Although data collection by BIFS has received research ethics approval, currently just twenty centres have research and development (R\&D) approval from their Trusts; the other five more centres are awaiting R\&D Trust approval. Outcome data (PN dependency status; complications; transplantation; death) are solicited at six-monthly intervals.

Between July 2005 and June 2008, 142 subjects (seventy-four males, sixty-eight females) have been registered. Median age at commencement of PN depended on the underlying diagnosis (see Table). In infants with congenital causes for IF, those with enteropathy were the oldest at the time of commencing PN, perhaps reflecting the difficulties in confirming the diagnosis in an uncommon disorder. The diagnoses in the miscellaneous category included bone marrow transplant, Crohn's disease and neoplasia, which accounts for the older age when starting PN, since the cause of IF in this group is acquired rather than congenital. This group also had the better prognosis, with few remaining on PN long term and none referred for ITx. Approximately $20 \%$ of registered patients were referred for small bowel transplant assessment, which may reflect a positive response to recommendations to involve a transplant centre when complications first arise. Patients with short bowel syndrome appeared to have the most adverse outcomes (death or ITx).

Table. Diagnosis, age and outcomes of patients registered with BIFS

\begin{tabular}{lcccrc}
\hline Main diagnosis & $n$ & $\begin{array}{c}\text { Median age at } \\
\text { start of PN }\end{array}$ & $\begin{array}{c}\text { Referred to BCH } \\
\text { for ITx assessment }\end{array}$ & $\begin{array}{c}\text { Discontinued } \\
\text { PN* }^{*}\end{array}$ & $\begin{array}{c}\text { Received } \\
\text { ITx* }\end{array}$ \\
\hline Short bowel syndrome & 99 & $4 \mathrm{~d}$ & 21 & 43 & 4 \\
Disorder of motility & 12 & $44 \mathrm{~d}$ & 4 & 4 & 88 \\
Enteropathy & 12 & $89 \mathrm{~d}$ & 3 & 0 & 1 \\
Miscellaneous & 19 & approx 1.5 years & 0 & 16 & 0 \\
\hline
\end{tabular}

BCH, Birmingham Children's Hospital; approx, approximately.

*Latest follow-up June 2008

The present interim report demonstrates that a national dataset for children with IF can be established through a cooperative effort on the part of the BSPGHAN representing paediatric gastroenterologists in the UK and with close working relationships with the British Association of Paediatric Surgeons. Recruitment has improved since June 2007 when lead investigators have been identified for each centre and administrative assistance has been provided by the IF Registry Administrator, but further work is needed to achieve a comprehensive registry. 